\title{
Spatial and Temporal Variations of Diurnal Ichthyofauna on Surf-Zone of São Francisco do Itabapoana Beaches, Rio de Janeiro State, Brazil
}

\author{
Marcelo Paes Gomes", Miguel Siano Cunha and Ilana Rosental Zalmon \\ Laboratório de Ciências Ambientais; Centro de Biociências e Biotecnologia; Universidade Estadual do Norte \\ Fluminense; Av. Alberto Lamego, 2000; 28013-600; ilana@uenf.br; Campos dos Goytacazes - RJ - Brazil
}

\begin{abstract}
Spatial and temporal variations of diurnal ichthyofauna and the environmental variables influences on its distribution were studied at the surf-zone of three beaches of São Francisco do Itabapoana, northern coast of Rio de Janeiro, Brazil. From August/1999 to August/2000, three beach seine hauls were made monthly, and environmental variables were recorded. A total number of 4,562 fishes $(74,155 \mathrm{~g})$ were sampled at the three beaches, where estuarine-dependent species prevailed (44\%), followed by marine (31\%), estuarine (19\%) and freshwater species $(3 \%)$. Species richness, number of individuals and wet weight were significantly higher at Gargaú, followed by Manguinhos and Barra do Itabapoana, respectively. Canonical Correspondence Analysis highlighted influences of the rivers flushing, salinity and plant abundance on the diurnal ichthyofauna distribution and dynamics of São Francisco do Itabapoana surf-zone.
\end{abstract}

Key words: Spatial and temporal variations; community ichthyofauna structure; surf-zone; environmental variables; north coast of Rio de Janeiro

\section{INTRODUCTION}

Estuaries and adjacent nearshore constitute important areas of coastal zone. Estuarine fish communities represent a combination of freshwater and marine species that live on the threshold of its distribution, other estuarine residents and migrants that reach the estuarine zone at spawning or feeding season (Maes et al, 1998; Cardona, 2000). With the nearshore coast, surf-zones and estuaries of tropical and temperate zones act as nursery, providing abundant food resources (Modde and Ross, 1981) and protection against predators (Anderson et al, 1977).

As an important beach modeler (Hoefel, 1998) and transporter of organic matter and nutrients between continent and sea (YañezAranciabia, 1978), the surf-zones support a widely fauna, mainly zooplankton and fishes (Maes et al, 1998). In fact, it has been observed that environmental complexity in terms of wave exposure and microhabitats formation, like seagrass and reefs, are related to composition and dynamics of surf-zone fish assemblages (Clark et al, 1996; Clark, 1997).

Surf-zone ichthyofauna are both seasonal and diel dynamic (Modde and Ross, 1981). Lasiak (1984) accepted that surf-zones concentrated a wide ichthyofauna, relatively high species richness, comparing to better-known environments, like

\footnotetext{
* Author for correspondence
} 
estuaries and shelf. However, a small number of species dominated numerically and were represented by juveniles (Naughton and Saloman, 1978; Monteiro-Neto et al, 1990; Ayizian and Hyndes, 1995; Nash and Santos, 1998).

Fisheries activity in northern coast of Rio de Janeiro is economically important, and has been considered the second major economy during 80's decade (IBAMA, 1986, 1991). The lack of studies concerning fauna surveys and biological aspects of fish assemblages in this region raises doubts about the conditions of those stocks. Being considered as nursery and growth site for many species, including economically important ones, it is necessary to study surf-zones of northern coast of Rio de Janeiro.

The comprehension of ichthyofauna dynamics for evaluation and management requires integrated analysis of physical, chemical and biological processes in spatial and temporal scales, due to adapted strategies of species life cycles to regional and seasonal variations (YAÑEZ-ARANCIBIA, 1978). The aim of this was perform an exploratory and comparative study on composition and structure of surf-zone fish assemblages on coast north of Rio de Janeiro at distinct beaches influenced by Itabapoana and Paraíba do Sul Rivers.

The hypothesis to be tested was:

$\mathrm{H} 1$ : spatial and temporal variations on composition and structure of São Francisco do Itabapoana surf-zone ichthyofauna are due to environmental variation of water temperature, salinity, $\mathrm{pH}$, plant abundance, pluviosity and flushing of Itabapoana and Paraíba do Sul Rivers.

\section{MATERIALS AND METHODS}

Sample stations were defined at Barra do Itabapoana beach $\left(21^{\circ} 17^{\prime} \mathrm{S}, 40^{\circ} 57^{\prime} \mathrm{W}\right)$, adjacent to Itabapoana River estuary, Gargaú beach $\left(21^{\circ} 37^{\prime} \mathrm{S}\right.$, $\left.41^{\circ} 05^{\prime} \mathrm{W}\right)$, adjacent to Paraíba do Sul River estuary and, Manguinhos beach $\left(21^{\circ} 23^{\prime} \mathrm{S}, 41^{\circ} 01^{\prime}\right.$ $\mathrm{W})$, intermediate between the referred estuaries (Fig. 1). Barra do Itabapoana e Gargaú stations were sampled in the closest surf-zone as possible to the estuaries. The north coast of Rio de Janeiro presents oligotrophic waters, influenced by Brazil current at north and South Atlantic Central Water (SACW) at south (Valentin and Monteiro-Ribas, 1993; Ekau and Knoppers, 1999), comprising almost $200 \mathrm{~km}$ of beaches. Barra do Itabapoana beach presents channels parallels to the shore.
Itabapoana River estuary is intensively modified, due to natural scavenging of the right margin and depositing on the left, contributing to constant morphological alterations of the outer estuary. Gargau beach is adjacent to Paraiba do Sul River estuary, presenting regularly deposits of matter consisted by plant detritus, like leaves and branches. Manguinhos beach represents a geomorphologic transition, exhibiting iron concressions. This beach regularly concentrates detached macroalgae.

Sampling was undertaken monthly, from August/1999 to August/2000, with a 25 x $3 \mathrm{~m}$ beach seine $(12 \mathrm{~mm}$ mesh, with a $2.5 \mathrm{~m} \mathrm{sac}, 9 \mathrm{~mm}$ mesh). At each site, three consecutive $50 \mathrm{~m}$ hauls were made, $1.5 \mathrm{~m}$ deep and parallel to the shore. The hauls followed the current direction using ballast attached to the net sac. All sampling were performed on the same day, during 7:00h to 13:00h in low tide peak.

For evaluation of environmental factors influence related to estuaries, the parameters considered were: mean flushing $\left(\mathrm{m}^{3} / \mathrm{s}\right)$ of Itabapoana and Paraíba do Sul Rivers, water temperature $\left({ }^{\circ} \mathrm{C}\right)$, salinity and $\mathrm{pH}$. Mean flushing $(\mathrm{m} 3 / \mathrm{s})$ data of Itabapoana and Paraíba do Sul Rivers represented month means from January/1990 to December/1999, and registered the nearest station flush of estuary (Itabapoana River- $21^{\circ} 12^{\prime} \mathrm{S}$; $41^{\circ} 27^{\prime} \mathrm{W}$ and Paraíba do Sul River - $21^{\circ} 45^{\prime} \mathrm{S}$; $\left.41^{\circ} 10^{\prime} \mathrm{W}\right)$.

Plant abundance at the beaches was determined by microhabitat formation (Clark et al, 1996). Plant material sampled in the seine were packed in $10 \mathrm{~kg}$ plastic sacks, estimating abundance as followed: 0 - no plant; 1 - < 50kg; 2 -60 a 100kg; 3 -110 a 150kg; 4 -160 a 200kg; 5 - > 200kg.

Fish species identification was based on Figueiredo and Menezes (1978; 1980), Menezes and Figueiredo (1980; 1985) and FAO (1978). Recorded data were treated as species richness and abundance (number of individuals and wet weight in g). Shannon diversity $\left(\mathrm{H}^{\prime}\right)$ was used to determine surf-zone ichthyofauna structure of São Francisco do Itabapoana beaches based on number of individuals (Zar, 1999).

Monofactorial ANOVA was used to test hypothesis that existed spatial and temporal variations on surfzone ichthyofauna of São Francisco do Itabapoana beaches (ZAR, 1999; $\mathrm{p} \leq 0.05$ ):

- among the three sites concerning physicochemical factors (water temperature, salinity, $\mathrm{pH}$ and plant abundance), biotic factors 
(species richness and abundance) and community structure (Shannon diversity);

- among 13 months sampling, concerning biotic factors of each beach.

For each significant different factor, StudentNewman-Keuls test (SNK; Zar, 1999; $\mathrm{p} \leq$ 0.05) was applied to determine differences between each beach and each of the 13 months sampled. Logarithmic transformation $(\log \mathrm{x})$ of the raw data was performed for all variables in order to validate assumptions for parametric analyses applied to the univariate and multivariate tests (Sokal and Rohlf, 1981).

Structural analysis of the assemblages was completed with the abundance data through canonical correspondence analysis (CCA). As selection criteria, species representing at least $2 \%$ of total number of individuals (except for Atherinella brasiliensis, second ranked and possibly a community structurer of Barra do Itabapoana beach) were used. Environmental matrix was composed by mean flushing of Itabapoana River $\left(\mathrm{m}^{3} / \mathrm{s}\right)$, mean flushing of Paraíba do Sul River $\left(\mathrm{m}^{3} / \mathrm{s}\right)$, water temperature $\left({ }^{\circ} \mathrm{C}\right)$, salinity, $\mathrm{pH}$ and plant abundance $(0-5)$. Only the significant variables through Monte Carlo test were selected.

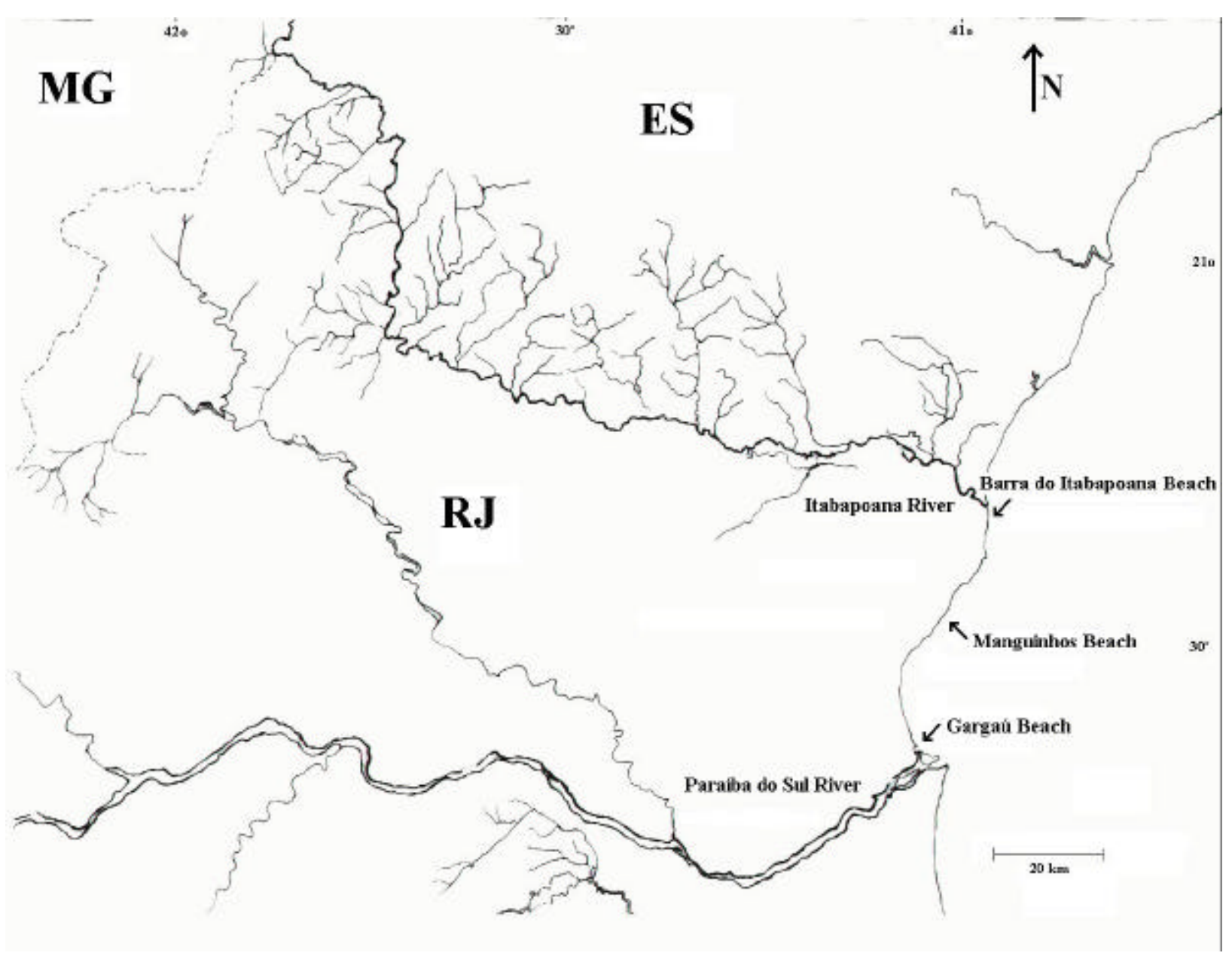

Figure 1 - Study area, showing the three sampling stations, located in São Francisco do Itabapoana, coast north of Rio de Janeiro: Barra do Itabapoana, Manguinhos and Gargaú. 


\section{RESULTS AND DISCUSSION}

Flushing patterns of Itabapoana and Paraíba do Sul Rivers presented a seasonal peak of higher flushing in January. Considering the ten years, Itabapoana River flushing was lower than Paraíba do Sul River in at least one order of magnitude (Fig. 2).

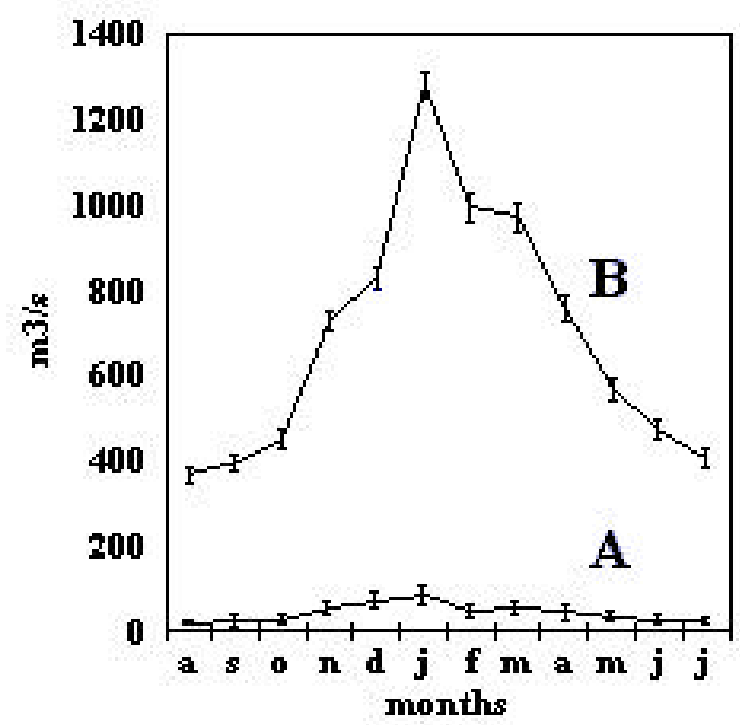

Figure 2 - Monthly mean flushing of Itabapoana (A) and Paraíba do Sul (B) Rivers from 1990 to 1999.

Water temperature showed increasing trends in summer months on the three beaches (Fig. 3A), with no significant differences among them. Manguinhos beach showed higher variation amplitude, between 20.2 and $34.4^{\circ} \mathrm{C}$ (mean $=24.8$ ${ }^{\circ} \mathrm{C}$ ). At Barra do Itabapoana (mean $=25 \cdot 2^{\circ} \mathrm{C}$ ) and Gargaú $\left(\right.$ mean $=23.9^{\circ} \mathrm{C}$ ) values higher then $30^{\circ} \mathrm{C}$ were not recorded, with lower ones around $20^{\circ} \mathrm{C}$ at both beaches.
Barra do Itabapoana presented low continental water influence, with higher values of salinity (Fig. 3B) and pH (Fig. 3C). Low Itabapoana River flushing and morphological alteration of the river mouth, due to migrating of sediment from right to the left margins and changes on the flow direction of the river channel, should be considered.

Seasonal variation of salinity at Manguinhos beach, with decreasing values on summer months (Fig. 3B), suggested that Paraíba do Sul River influence reached this beach in summer, when flushing was significantly higher.

Gargaú beach was clearly influenced by Paraíba do Sul River estuarine zone. Low salinity values (Fig. 3B) and increased plant abundance (leaves and branches) in summer months (Fig. 3D) occurred on increased flushing season (Fig. 2), showing this influence.

Manguinhos presented a high amount of detached macroalgae (Fig. 3D). Higher peaks were reached in summer and winter months. These algae, after detachment from the substrate by storms and/or strong wave actions could be carried from deeper regions of the shelf and concentrated in the nearshore, as verified by CLARK et al (1996). Significant differences $(\mathrm{p}<0.05)$ were observed for salinity and plant abundance among the three beaches (Table 1).

A total number of 68 fish species were registered on the surf-zone of São Francisco do Itabapoana (Table 2). Estuarine-dependent species prevailed (44\%), followed by marine (28\%), estuarine (22\%) and freshwater $(6 \%)$ species. Gargaú beach presented wider variety of species in terms of habitat preference, being the only station where freshwater species were recorded (Table 2). Nevertheless, none of them was abundant. 

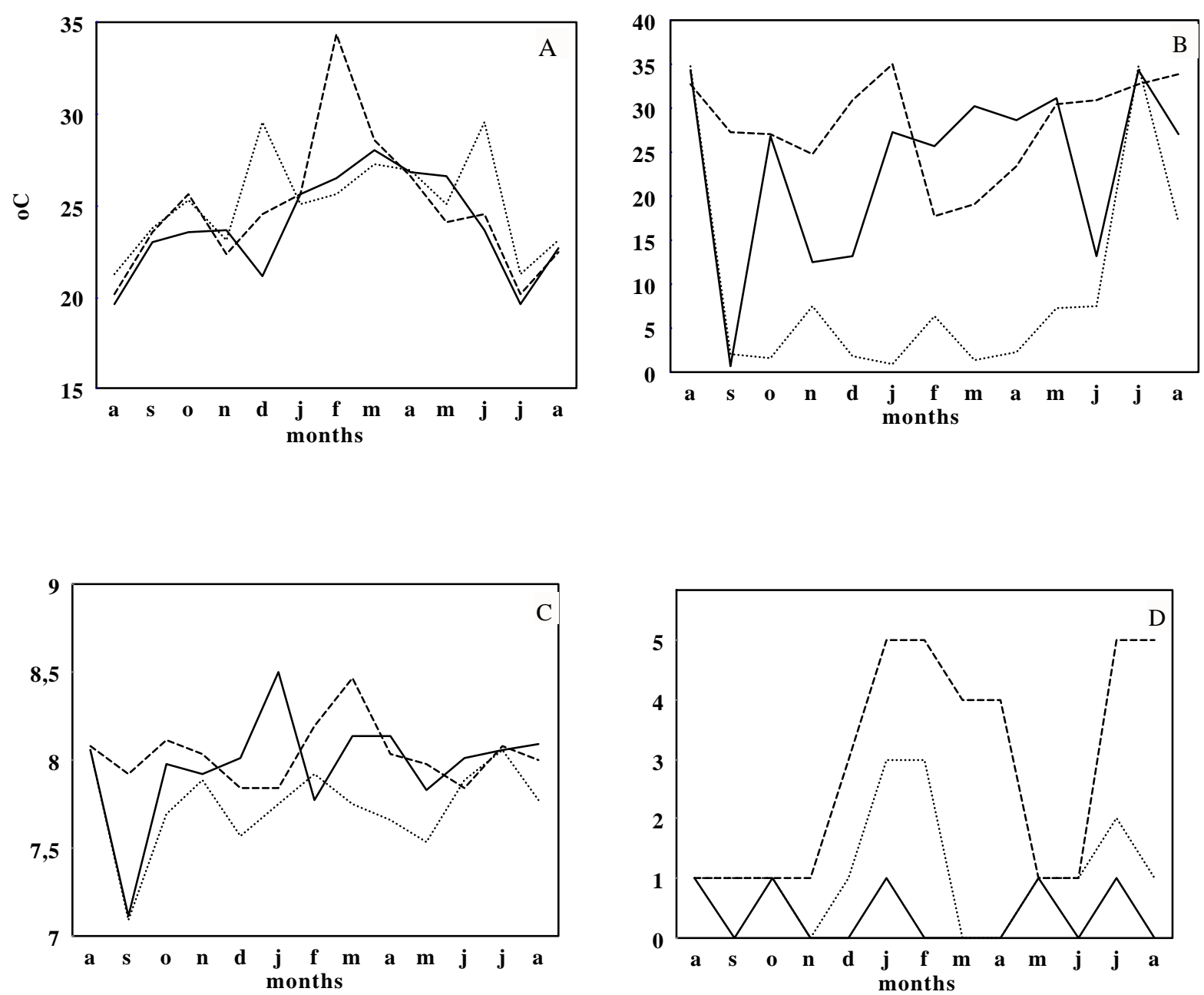

Figure 3 - Temporal variation of water temperature (A), salinity (B), $\mathrm{pH}(\mathrm{C})$ and plant abundance (D, see scaling in materials and methods) in Barra do Itabapoana (line), Manguinhos (trace) and Gargaú (minor trace) surf-zones from Aug/99 to Aug/00.

Table 1 - F-values and significant differences of monofactorial ANOVA among Barra do Itabapoana (BI), Manguinhos (M) e Gargaú (G) beaches and among study period (Aug/99 to Aug/00). Post hoc comparisons performed using Student-Newman-Keuls (SNK) test. Obs: *p $<0.05 ; * * \mathrm{p}<0.01 ; * * * \mathrm{p}<0.001$

\begin{tabular}{|c|c|c|c|c|c|}
\hline \multirow[t]{2}{*}{ Beaches $(n=117)$} & \multirow[t]{2}{*}{$\mathbf{F}$} & \multirow[t]{2}{*}{$\mathbf{p}$} & \multicolumn{3}{|c|}{ SNK } \\
\hline & & & BI X M & M X G & BI X G \\
\hline Water temperature & 0.592 & & & & \\
\hline Salinity & 12.960 & $* * *$ & & $* * *$ & $* * *$ \\
\hline $\mathrm{PH}$ & 2.051 & & & & \\
\hline Plant abundance & 13.144 & $* * *$ & $* * *$ & $* *$ & \\
\hline Species richness & 7.063 & $* * *$ & $* * *$ & & $* * *$ \\
\hline Number of individuals & 4.167 & $*$ & & $*$ & $*$ \\
\hline Wet weight & 8.814 & $* * *$ & $* *$ & $* *$ & \\
\hline Shannon diversity & 2.707 & & & & \\
\hline Sample period & $\mathrm{F}$ & $\mathrm{p}$ & & SNK & \\
\hline
\end{tabular}


(Cont. Table 1)

\begin{tabular}{|c|c|c|c|c|c|}
\hline \multicolumn{6}{|l|}{ Barra do Itabapoana $(n=39)$} \\
\hline Species richness & 4.150 & $* *$ & Sep, Nov & $>$ & May, Jun, Aug/00 \\
\hline Number of individuals & 1.538 & & & & \\
\hline Wet weight & 2.357 & $*$ & Nov & $>$ & $\begin{array}{c}\text { Aug/99, May, Jun, } \\
\text { Jul, Aug/00 }\end{array}$ \\
\hline \multicolumn{6}{|l|}{ Manguinhos $(n=39)$} \\
\hline Species richness & 3.829 & $* *$ & Feb, May, Jun & $>$ & Aug/99, Mar, Jul/00 \\
\hline Number of individuals & 9.180 & $* * *$ & $\mathrm{Feb}$ & $>$ & other months \\
\hline Wet weight & 6.817 & $* * *$ & Feb & $>$ & other months \\
\hline \multicolumn{6}{|l|}{ Gargaú (n = 39) } \\
\hline Species richness & 4.671 & $* * *$ & $\begin{array}{c}\text { Aug/99, Jan, } \\
\text { Aug/00 }\end{array}$ & $>$ & May, Jun/00 \\
\hline Number of individuals & 3.441 & $* * *$ & Jan, Feb & $>$ & other months \\
\hline Wet weight & 5.900 & $* * *$ & Feb & $>$ & other months \\
\hline
\end{tabular}

Table 2 - Fish species, absolute and relative individuals abundance and preferential habit (F - Freshwater; E estuarine; ED - estuarine-dependent; M - marine) registered at surf-zone of São Francisco do Itabapoana beaches. Fish habits were compiled from 1 - FAO (1978), 2, 3, 8 - Figueiredo and Menezes (1978, 1980, 2000), 4, 5 Menezes and Figueiredo (1980, 1985), 6 - Gianinni (1994) and 7 - Pessanha et al. (2000).

\begin{tabular}{|c|c|c|c|c|c|c|c|c|c|c|}
\hline \multirow[t]{2}{*}{ Taxon } & \multirow[t]{2}{*}{ Habit } & \multirow[t]{2}{*}{ Reference } & \multicolumn{4}{|c|}{$\begin{array}{cc}\text { Barra do } \\
\text { All beaches } \\
\text { Itabapoana }\end{array}$} & \multicolumn{2}{|c|}{ Manguinhos } & \multicolumn{2}{|c|}{ Gargaú } \\
\hline & & & $\mathbf{n}$ & $\%$ & $\mathbf{n}$ & $\%$ & $\mathbf{n}$ & $\%$ & $\mathbf{n}$ & $\%$ \\
\hline Sciadeichthys luniscutis & $\mathrm{ED}$ & $2,6,7$ & 1136 & 24.9 & 20 & 4.2 & 586 & 34.3 & 530 & 22.2 \\
\hline Cathorops spixii & $\mathrm{ED}$ & $1,2,7$ & 795 & 17.4 & - & - & 181 & 10.6 & 614 & 25.8 \\
\hline Conodon nobilis & M & 1,4 & 353 & 7.7 & 44 & 9.3 & 174 & 10.2 & 135 & 5.7 \\
\hline Netuma barba & $\mathrm{E}$ & 2,7 & 291 & 6.4 & 100 & 21.2 & 5 & $<2.0$ & 186 & 7.8 \\
\hline Hexanematichthys grandoculis & $\mathrm{E}$ & 2 & 201 & 4.4 & 23 & 4,9 & 25 & $<2.0$ & 153 & 6.4 \\
\hline Stellifer brasiliensis & ED & 1,4 & 199 & 4.4 & 2 & $<2.0$ & 183 & 10.7 & 14 & $<2.0$ \\
\hline Stellifer stellifer & M & 1,4 & 183 & 4.0 & 3 & $<2.0$ & 64 & 3.7 & 116 & 4.9 \\
\hline Anchoa januaria & E & 2 & 182 & 3.9 & 35 & 7.4 & 42 & 2.5 & 105 & 4.4 \\
\hline Genidens genidens & $\mathrm{E}$ & 2,7 & 175 & 3.8 & 17 & 3.6 & 3 & $<2.0$ & 155 & 6.5 \\
\hline Polydactylus virginicus & M & $1,5,6$ & 169 & 3.7 & 9 & $<2.0$ & 87 & 5.1 & 73 & 3.1 \\
\hline Trachinotus goodei & M & 1,4 & 120 & 2.6 & 41 & 8.7 & 33 & $<2.0$ & 46 & $<2.0$ \\
\hline Trachinotus carolinus & M & 1,4 & 98 & 2.1 & 36 & 7.6 & 32 & $<2.0$ & 30 & $<2.0$ \\
\hline \multicolumn{11}{|l|}{ Continue } \\
\hline Menticirhus littoralis & ED & 1,4 & 86 & 2.0 & 34 & 7.2 & 50 & 2.9 & 2 & $<2.0$ \\
\hline Atherinella brasiliensis & $\mathrm{E}$ & 2 & 53 & $<2.0$ & 44 & 9.3 & 5 & $<2.0$ & 4 & $<2.0$ \\
\hline Stellifer rastrifer & ED & 1,4 & 48 & $<2.0$ & 3 & $<2.0$ & 12 & $<2.0$ & 33 & $<2.0$ \\
\hline Cetengraulis edentulus & $\mathrm{ED}$ & 2 & 42 & $<2.0$ & - & - & 1 & $<2.0$ & 41 & $<2.0$ \\
\hline Anchoviella lepidontostole & $\mathrm{ED}$ & 2 & 40 & $<2.0$ & 14 & 3.0 & 12 & $<2.0$ & 14 & $<2.0$ \\
\hline Caranx crysos & M & 1,4 & 36 & $<2.0$ & 10 & 2.1 & 11 & $<2.0$ & 15 & $<2.0$ \\
\hline Mugil gaimardianus & $\mathrm{ED}$ & 1,5 & 35 & $<2.0$ & 14 & 3.0 & 11 & $<2.0$ & 10 & $<2.0$ \\
\hline Ophioscion punctatissimus & M & 1,4 & 25 & $<2.0$ & - & - & 24 & $<2.0$ & 1 & $<2.0$ \\
\hline Micropogonias furnieri & ED & 1,4 & 22 & $<2.0$ & - & - & 21 & $<2.0$ & 1 & $<2.0$ \\
\hline Trachinotus falcatus & M & 1,4 & 22 & $<2.0$ & 1 & $<2.0$ & 14 & $<2.0$ & 7 & $<2.0$ \\
\hline Stellifer $\mathrm{sp}$ & ED & 1,4 & 21 & $<2.0$ & - & - & 20 & $<2.0$ & 1 & $<2.0$ \\
\hline Hiporhampus unifasciatus & E & 2 & 17 & $<2.0$ & - & - & 15 & $<2.0$ & 2 & $<2.0$ \\
\hline Paralichthys brasiliensis & E & 1,8 & 16 & $<2.0$ & 3 & $<2.0$ & 2 & $<2.0$ & 11 & $<2.0$ \\
\hline Oligoplities saliens & $\mathrm{ED}$ & 1,4 & 15 & $<2.0$ & - & - & 8 & $<2.0$ & 7 & $<2.0$ \\
\hline Mugil sp2 & ED & 1,5 & 14 & $<2.0$ & - & - & - & - & 14 & $<2.0$ \\
\hline Larimus breviceps & ED & 1,4 & 13 & $<2.0$ & 1 & $<2.0$ & 12 & $<2.0$ & - & - \\
\hline Strongylura marina & $\mathrm{E}$ & 2 & 13 & $<2.0$ & 4 & $<2.0$ & 1 & $<2.0$ & 8 & $<2.0$ \\
\hline Umbrina coroides & ED & 1,4 & 13 & $<2.0$ & - & - & 13 & $<2.0$ & - & - \\
\hline Lagocephalus laevigatus & M & 1,8 & 12 & $<2.0$ & - & - & 9 & $<2.0$ & 3 & $<2,0$ \\
\hline
\end{tabular}




\begin{tabular}{|c|c|c|c|c|c|c|c|c|c|c|}
\hline Chloroscombrus crysurus & $\mathrm{M}$ & 1,4 & 10 & $<2.0$ & - & - & 6 & $<2.0$ & 4 & $\overline{<2,0}$ \\
\hline Selene vomer & ED & 1,4 & 9 & $<2.0$ & 1 & $<2.0$ & 6 & $<2.0$ & 2 & $<2,0$ \\
\hline Centropomus parallelus & ED & 1,3 & 8 & $<2.0$ & 3 & $<2.0$ & 5 & $<2.0$ & - & \\
\hline Mugil incilis & ED & 1,5 & 7 & $<2.0$ & 1 & $<2.0$ & 6 & $<2.0$ & - & \\
\hline Sphoeroides testudineus & ED & 8 & 7 & $<2.0$ & - & - & 5 & $<2.0$ & 2 & $<2,0$ \\
\hline Bagre bagre & M & 1,2 & 6 & $<2.0$ & - & - & 3 & $<2.0$ & 3 & $<2,0$ \\
\hline Menticirhus americanus & ED & 1,4 & 6 & $<2.0$ & - & - & 6 & $<2.0$ & - & \\
\hline Mugil platanus & ED & 1,5 & 6 & $<2.0$ & - & - & - & - & 6 & $<2,0$ \\
\hline Trichiurus lepturus & ED & 1,8 & 6 & $<2.0$ & - & - & 1 & $<2.0$ & 5 & $<2,0$ \\
\hline Achirus sp & $\mathrm{E}$ & 1 & 5 & $<2.0$ & - & - & - & - & 5 & $<2,0$ \\
\hline Boridia grossidens & M & 4 & 5 & $<2.0$ & - & - & 4 & $<2.0$ & 1 & $<2,0$ \\
\hline Gobioides braussonnetti & $\mathrm{E}$ & 5 & 5 & $<2.0$ & 2 & $<2.0$ & - & - & 3 & $<2,0$ \\
\hline Isopisthus parvippinis & ED & 1,4 & 5 & $<2.0$ & - & - & - & - & 5 & $<2,0$ \\
\hline Gobionellus oceanicus & $\mathrm{E}$ & 5 & 3 & $<2.0$ & - & - & - & - & 3 & $<2,0$ \\
\hline Caranx latus & M & 1,4 & 2 & $<2.0$ & 1 & $<2.0$ & - & - & 1 & $<2.0$ \\
\hline Chaetodipterus faber & M & 1,5 & 2 & $<2.0$ & - & - & 2 & $<2.0$ & - & \\
\hline Epinephelus niveatus & ED & 1,3 & 2 & $<2.0$ & - & - & 2 & $<2.0$ & - & \\
\hline Eugerres brasilianus & ED & 1,4 & 2 & $<2.0$ & 2 & $<2.0$ & - & - & - & \\
\hline Odontognathus mucronatus & M & 2 & 2 & $<2.0$ & - & - & 1 & $<2.0$ & 1 & $<2.0$ \\
\hline Prionotus punctatus & ED & 1,3 & 2 & $<2.0$ & - & - & 2 & $<2.0$ & - & \\
\hline Salminus maxillosus & $\mathrm{F}$ & - & 2 & $<2.0$ & - & - & - & - & 2 & $<2.0$ \\
\hline Trachinotus sp & ED & 1,4 & 2 & $<2.0$ & 2 & $<2.0$ & - & - & - & \\
\hline Archosargus probatocephalus & ED & 1,4 & 1 & $<2.0$ & - & - & - & - & 1 & $<2.0$ \\
\hline Cychlasoma fasciatum & $\mathrm{F}$ & - & 1 & $<2.0$ & - & - & - & - & 1 & $<2.0$ \\
\hline Cynoscion microlepidotus & ED & 1,4 & 1 & $<2.0$ & - & - & - & - & 1 & $<2.0$ \\
\hline Cynoscion virescens & $\mathrm{ED}$ & 1,4 & 1 & $<2.0$ & - & - & - & - & 1 & $<2.0$ \\
\hline Elops saurus & ED & 1,2 & 1 & $<2.0$ & - & - & - & - & 1 & $<2.0$ \\
\hline Gymnotus carapo & $\mathrm{F}$ & - & 1 & $<2.0$ & - & - & - & - & 1 & $<2.0$ \\
\hline Lobotes surinamensis & ED & 1,4 & 1 & $<2.0$ & - & - & - & - & 1 & $<2.0$ \\
\hline Continue & & & & & & & & & & \\
\hline Macrodon ancylodon & ED & 1,4 & 1 & $<2.0$ & - & - & - & - & 1 & $<2.0$ \\
\hline Mugil sp & $\mathrm{ED}$ & 1,5 & 1 & $<2.0$ & 1 & $<2.0$ & - & - & - & \\
\hline Orthopristis ruber & $\mathrm{M}$ & 1,4 & 1 & $<2.0$ & - & - & 1 & $<2.0$ & - & \\
\hline Pimelodidae & $\mathrm{F}$ & 1 & 1 & $<2.0$ & - & - & - & - & 1 & $<2.0$ \\
\hline Selene setapinnis & $\mathrm{M}$ & 1,4 & 1 & $<2.0$ & - & - & 1 & $<2.0$ & - & \\
\hline Symphurus sp & $\mathrm{M}$ & 1,8 & 1 & $<2.0$ & - & - & 1 & $<2.0$ & - & \\
\hline Talassophorine nattereri & M & 2 & 1 & $<2.0$ & 1 & $<2.0$ & - & - & - & \\
\hline Trachinotus marginatus & $\mathrm{M}$ & 1,4 & 1 & $<2.0$ & - & - & - & - & 1 & $<2.0$ \\
\hline Total & & & 4564 & 100 & 472 & 100 & 1708 & 100 & 2384 & 100 \\
\hline
\end{tabular}

Other studies in Brazil (Monteiro-Neto et al., 1990; Pessanha et al, 2000) and other countries (Clark et al., 1994) also considered freshwater species as rare and few abundant on estuarine beach adjacencies. Manguinhos and Gargaú beaches registered increased variety of species in summer months (Fig. 4A), due to the combination of marine, estuarine and estuarine-dependent fishes. Seagrass provided shelter and food resources (Lenanton et al, 1982). Those conditions could affect fish abundance, corroborated by the low yield in Barra do Itabapoana, comparing to the other stations. It was expected that Barra do Itabapoana ichthyofauna would be more similar to Gargaú (due to the proximity of estuaries).
Nevertheless, the former registered lower species richness and abundance than the latter (fig. 4). At Barra do Itabapoana, channel formations parallels to the shore might be relevant disturbances for surf-zone ichthyofauna. Harvey (1998) related channel formations to lower fish abundances, due to increased deep, facilitating presence of predators on surf-zones. A total number of 4,564 fishes were registered on surf-zone of São Francisco do Itabapoana, with 472 individuals in Barra do Itabapoana, 1,708 in Manguinhos and 2,384 in Gargaú (Table 2). The ichthyofauna consisted of juveniles of many species, most of then non-dominants. This pattern has been largely described at other beaches (Giannini, 1994; 
Ayivazian and Hyndes, 1995; Clark, 1997; Pessanha et al., 2000; Gaelzer, 2000). Fishes commonly use surf-zones as nursery and growth grounds, profiting by food abundance and protection against predation (Lasiak, 1984). Barra do Itabapoana showed similar proportions of marine, estuarine and estuarine-dependent species, while at Manguinhos and Gargaú estuarinedependent species prevailed.

Manguinhos beach presented the highest mean species richness (Fig. 4A) varying from 3.7 to 14.0 (mean $=8.0 \mathrm{spp}$ ), followed by Gargaú beach (from 3 to 13.2 ; mean $=7.4 \mathrm{spp}$ ). Barra do Itabapoana beach registered the lowest species richness, varying from 1.0 to 7.5 (mean $=4.0 \mathrm{spp})$. Barra do Itabapoana and Gargaú beaches showed trends of increasing species richness on spring-summer and summer months, respectively, while no seasonality was evidenced in Manguinhos beach.

Gargaú beach presented the highest mean abundance of number of individuals (Fig. 4B), from 4 to 182 (mean $=61$ individuals), followed by Manguinhos beach (4 to 212 individuals; mean $=46$ individuals). These stations showed increasing abundance on summer months, especially January and February/2000. The lowest abundances were recorded in Barra do Itabapoana, from 1 to 28 individuals (mean $=12$ inds).
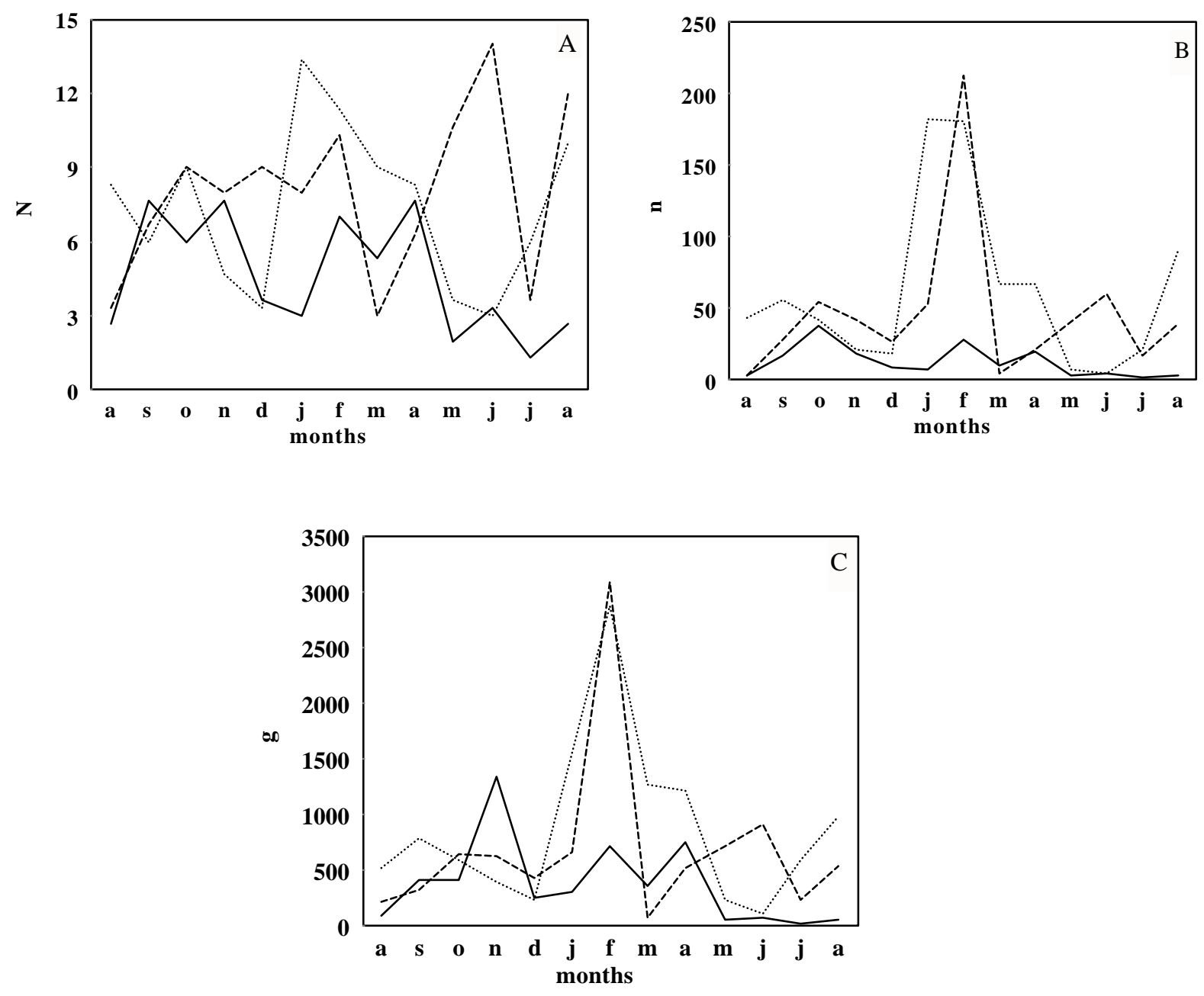

Figure 4 - Temporal variation of the means of species richness (A), number of individuals (B) and (line), Manguinhos (trace) and Gargaú (minor trace) surf-zones from Aug/99 to Aug/00. 
The highest mean abundance of wet weight was also registered in Gargaú beach (fig. 4C), varying from 110.6 to $2862.4 \mathrm{~g}$ (mean $=869.9 \mathrm{~g}$ ), followed by Manguinhos beach $(65.5$ a $3089.0 \mathrm{~g}$; mean = $688.3 \mathrm{~g}$ ). Trends of increasing abundance on summer months were also observed, especially in February/2000, with peaks higher than $2500 \mathrm{~g}$. The lowest weight abundance was registered in Barra do Itabapoana, varying from 17.3 to $1342.0 \mathrm{~g}$ (mean $=370.5 \mathrm{~g})$.

Species richness and abundance (number of individuals and wet weight) presented statistically significant spatial and temporal variations (Table 2). In Brazilian estuaries, high abundances of catfish (Ariidae) were common, comprising almost $8.0 \%$ of the total catching on Sepetiba Bay, southern Rio de Janeiro State (Pessanha et al, 2000). Godefroid (1996) recorded a similar percentage in Pontal do Sul beach, towards Paranagua estuarine complex, Paraná State.

In this study, catfish comprised more than $57.0 \%$ of total catch and at least one species dominated in each beach: $N$. barba in Barra do Itabapoana; $S$. luniscutis in Manguinhos and C. spixii and $S$. luniscutis in Gargaú. Catfishes were more associated to estuary adjacencies. In Manguinhos beach, only $C$. spixii and $S$. luniscutis were constant. These species, especially the second one, has been commonly caught by artisanal fishermen. GOMES et al (2001) found $S$. luniscutis five miles away from the shore. Other species, as $G$. genidens, $H$. grandoculis and $N$. barba were recorded more abundantly and constantly in Gargaú beach.

Shannon diversity values were not significantly different among the three stations (Table 1), varying mostly from 1.0 to 2.5 at all stations, with higher values especially in Manguinhos beach (Fig. 5). Declining diversity on summer months in Manguinhos and Gargaú beaches was due to the dominance of C. spixii in January/2000 (Gargaú) and S. luniscutis in February/2000 (Manguinhos and Gargaú). Barra do Itabapoana beach presented opposite trends of diversity on summer months, declining on winter months due to the low yield in this period.

The environmental factors selected after Monte Carlo Permutation test were salinity, Itabapoana River mean flushing, Paraíba do Sul (PS) River mean flushing and plant abundance.

The two first canonical axes explained $77.4 \%$ of total variance concerning species/environment relation. Monte Carlo test attested the significance of the two canonical axes at $95 \%$ level. The first canonical axis explained $41.4 \%$ of total variance. Itabapoana River flushing was positively correlated to axis I, while PS River flushing correlated negatively to this axis (Table 3). Second canonical axis explained $36,0 \%$ of total variance. Salinity and plant abundance were negatively correlated to axis II (Table 3).

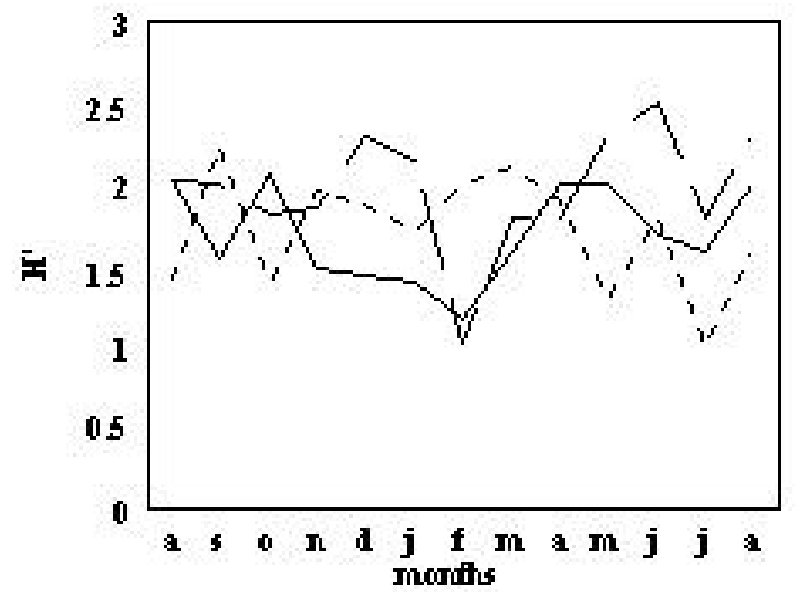

Figure 5 - Temporal variation of Shannon diversity on Barra do Itabapoana (line), Manguinhos (trace) and Gargaú (minor trace) surf-zones from Aug/99 to Aug/00.

Table 3 - Canonical Correspondence Analysis eigenvalues, percentage variance and environment/axes inter set correlations.

\begin{tabular}{|c|c|c|}
\hline & Axis I & Axis II \\
\hline Eigenvalues & 0.129 & 0.112 \\
\hline $\begin{array}{l}\text { Percentage variance } \\
\text { of species }\end{array}$ & 10.2 & 8.8 \\
\hline $\begin{array}{l}\text { of species-environment } \\
\text { relation }\end{array}$ & 41.4 & 36.0 \\
\hline $\begin{array}{l}\text { Species-environment } \\
\text { correlations }\end{array}$ & 0.726 & 0.713 \\
\hline Inter-set correlations & & \\
\hline Salinity & 0.3834 & -0.8032 \\
\hline Itabapoana River flushing & 0.8221 & 0.4257 \\
\hline $\begin{array}{l}\text { Paraíba do Sul River } \\
\text { flushing }\end{array}$ & -0.7907 & 0.5651 \\
\hline Plant abundance & -0.2862 & -0.6094 \\
\hline
\end{tabular}

CCA highlighted four distinct groups of species, each one related to an environmental factor (Fig. 6). Group I, associated to PS River flushing, comprised A. januaria and the catfishes G. genidens, $H$. grandoculis and $N$. barba, 
typically estuarine species, negatively correlated to axis I. The inclusion of Gargaú species in this group showed estuarine influence species distribution. Group II, associated to Itabapoana River flushing, clustered A. brasiliensis, $T$. carolinus and $T$. goodei, positively correlated to axis I. However, only A. brasiliensis was an estuarine species. T. carolinus and T. goode $i$ were known as surf-zone resident species (Ross et al, 1987; Giannini, 1994). Only Barra do Itabapoana beach samples were recorded in this group. Group III, related to salinity, was composed by marine (C. nobilis and $P$. virginicus) and estuarinedependent (M. littoralis and $S$. brasiliensis) species. These species were regular when saline waters prevailed. Group IV, related to plant abundance, joined the catfishes $C$. spixii and $S$. luniscutis, and $S$. stellifer. This group was negatively correlated to axis II and positively related to Group I, led by Paraíba do Sul River flushing. All species were estuarine-dependent. This group recorded Manguinhos and Gargaú samples related to higher plant abundances. $S$. stellifer was numerically abundant in Manguinhos, especially during maximum peaks of seagrass abundance. Its diet was based on crustaceans (Giannini, 1994) that possibly composed detached seagrass fital (Robertson and Lenanton, 1984) in Manguinhos.

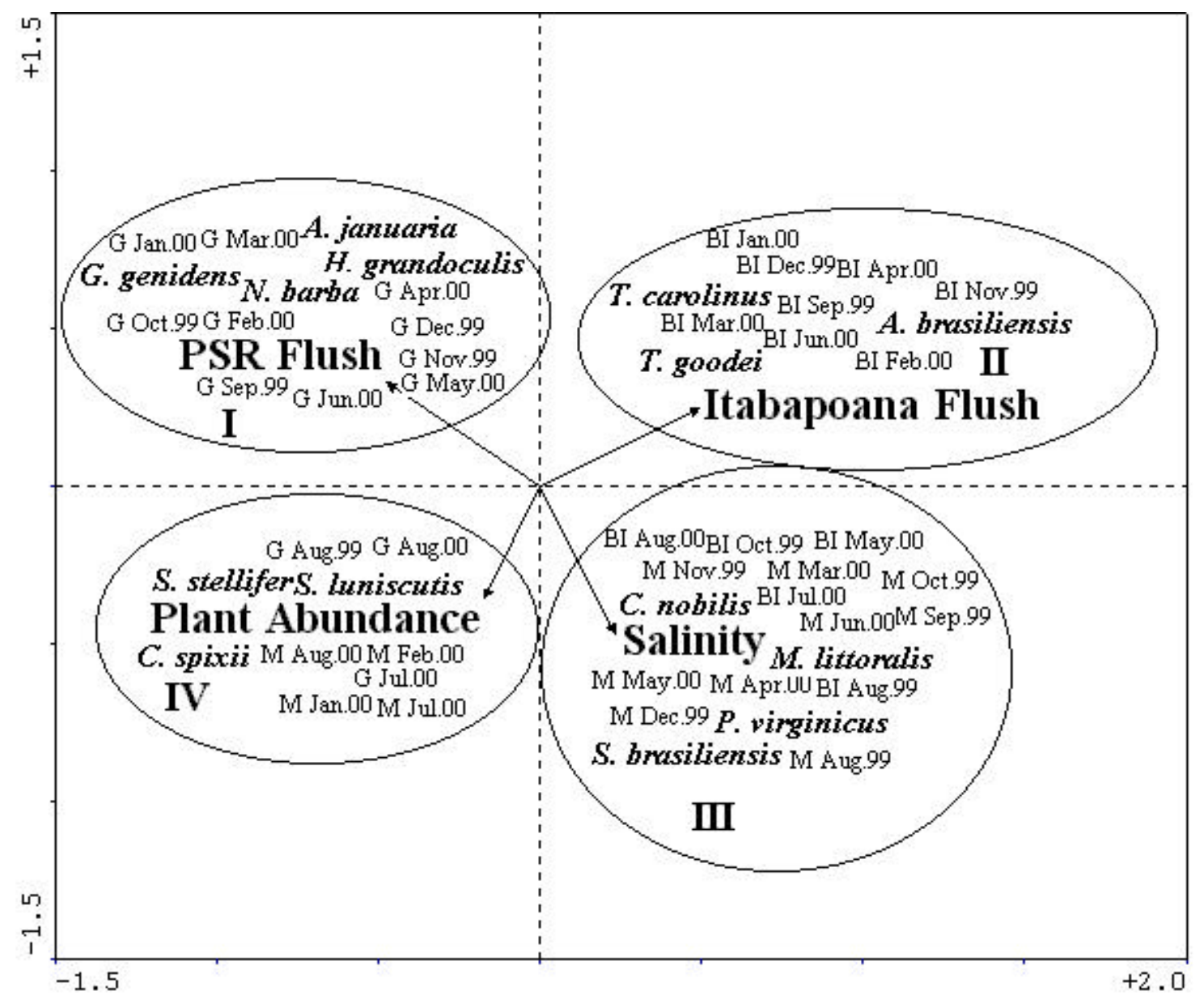

Figure 6 - Canonical correspondence analysis factorial diagram showing environmental influences on species and samples distribution of Barra do Itabapoana (BI), Manguinhos (M) and Gargaú (G) beaches from Aug/99 to Aug/00.

Obs.: PSR Flush = Paraíba do Sul River Flushing; Itabapoana Flush = Itabapoana River Flushing 
Both species of catfish would be related to increasing plant abundance in Gargaú, which has been used as shelter for young-of-the-year, seeking better chances of recruitment success. Anderson et al and Araújo et al (1977) suggested that surf-zone studies were important for the management of this environment, due to their use by juveniles of many fish species as nursery and growth grounds.

This study showed that most commercially valuable species were not numerically abundant in surf-zone of São Francisco do Itabapoana beaches, in accordance with IBAMA (1986, 1991). However, it was possible to generate information about potential preys of those species. More studies are necessary about trophic relations concerning shelf, estuaries and surf-zone ichthyofauna of northern coast of Rio de Janeiro.

\section{ACKNOWLEGMENTS}

We thank to Mr. Antônio Carlos Oliveira Pessanha; Alexandre Gomes Ferreira, Luis Ricardo Gaelzer, Vicente Vieira Faria; André Machado, Tércio Abreu and Thiago Parente. This project was supported by FENORTE.

\section{RESUMO}

Variações espaço-temporais da ictiofauna diurna e a influência de variáveis ambientais em sua distribuição foram estudadas na zona de arrebentação de três praias no Município de São Francisco do Itabapoana, costa norte do Rio de Janeiro. Entre agosto/1999 e agosto/2000 foram realizados mensalmente três arrastos com rede "picaré" e obtidos valores de variáveis ambientais em Barra do Itabapoana $\left(21^{\circ} 17^{\prime} \mathrm{S}, 40^{\circ} 57^{\prime} \mathrm{W}\right)$, adjacente ao estuário do rio Itabapoana, Gargaú $\left(21^{\circ} 37^{\prime} \mathrm{S}, 41^{\circ} 05^{\prime} \mathrm{W}\right)$, adjacente ao estuário do rio Paraíba do Sul, e Praia de Manguinhos (21 $23^{\circ}$ S, $41^{\circ} 01^{\prime} \mathrm{W}$ ), intermediária. Um total de 4.652 peixes $(74.155 \mathrm{~kg})$ foi capturado nas três praias, com predomínio de espécies estuarino-dependentes (44\%), seguidas por espécies marinhas (31\%), estuarinas (19\%) e dulcícolas (6\%). Valores de riqueza de espécies de peixes, número de indivíduos e peso úmido diferiram significativamente entre as praias, sendo superiores em Gargaú, seguida por Manguinhos e Barra do Itabapoana, respectivamente. A análise de correspondência canônica confirmou a influência das vazões de ambos os rios, da salinidade e da abundância de material vegetal na distribuição e dinâmica da ictiofauna diurna da zona de arrebentação de São Francisco do Itabapoana.

\section{REFERENCES}

Araújo, F. G.; Cruz-Filho, A. G.; Azevedo, M. C. C.; Santos, A. C. A. and Fernandes, L. A. M. (1997), Estrutura da Comunidade de Peixes Jovens da Margem Continental da Baía de Sepetiba, RJ. Acta Biol. Leopol., 19 : (1), 61-83.

Anderson Jr., W. D.; Dias, J. K.; Dias, R. K.; Cupka, D. M. and Chamberlain, N. A. (1977), The Macrofauna of the Surf Zone Off Folly Beach, South Carolina. NOAA Technical Report NMFS SSRF-704. 24 pp.

Ayvzian, S. G. and Hyndes, G. A. (1995), Surf-Zone Fish Assemblages in South-Western Australia: Do Adjacent Nearshore Habitats and the Warm Leeuwin Current Influence the Characteristics of the Fish Fauna? Mar. Biol., 122, 527-536.

Cardona, L. (2000), Effects of Salinity on the Habitat Selection and Growth Performance of Mediterranean Flathead Grey Mullet Mugil cephalus (Ostechthyes, Mugilidae). Est. Coast. Shelf Sci., 50, 727-737

Clark, B. M. (1997), Variation in Surf-Zone Fish Community Structure Across a Wave Exposure Gradient. Est. Coast. Shelf Sci., 44, 659-674.

Clark, B. M.; Bennett, B. A. and Lamberth, S. J. (1994), A Comparison of the Ichthyofauna of Two Estuaries and their Adjacent Surf Zones, With An Assessment of the Effects of Beach Seining of the Nursery Function of Estuaries for fish. S. Afr. Mar. Sci., 14, 121-131.

Clark, B. M.; Bennett, B. A. and Lamberth, S. J. (1996), Factors Affecting Spatial Variability in Seine Net Catches of Fish in The Surf Zone of False Bay, South Africa. Mar. Ecol. Prog. Ser., 131, 17-34.

Ekau, W. and Knoppers, B. (1999), An Introduction to the Pelagic System of the North-East and East Brazilian Shelf. Arch. Fish. Mar. Res., 47 : (2/3), 113-132.

FAO (1978), Species Identification Sheets for Fisheries Purposes: Western Central Atlantic (FA 31). 6 v. Roma : W. Fisher.

Figueiredo, J. L. and Menezes, N. A. (1978), Manual de Peixes Marinhos do Sudeste do Brasil. II Teleostei (1). Museu de Zoologia da Universidade de São Paulo, São Paulo. 110 pp.

Figueiredo, J. L. and Menezes, N. A. (1980), Manual de Peixes Marinhos do Sudeste do Brasil. III Teleostei (2). Museu de Zoologia da Universidade de São Paulo, São Paulo. 90 pp. 
Figueiredo, J. L. and Menezes, N. A. (1980), Manual de Peixes Marinhos do Sudeste do Brasil. VI Teleostei (5). Museu de Zoologia da Universidade de São Paulo, São Paulo, 116 pp.

Gaelzer, L. R. (2000), Influência do Grau de Exposição Às Ondas sobre A Estrutura da Comunidade Ictiofaunística na Zona de Arrebentação da Prainha, Arraial do Cabo, RJ. M.Sc Thesis, State University of Norte Fluminense, Campos. 67 pp.

Gianinni, R. (1994), Estrutura da Comunidade de Peixes da Zona de Arrebentação de Praias Arenosas do Litoral do Estado de São Paulo, BR. PhD Thesis, Instituto Oceanográfico, University of São Paulo, São Paulo, Brazil. 139 pp.

Godefroid, R. S. (1996), Estrutura Populacional da Ictiofauna da Zona de Arrebentação da Praia do Pontal do Sul, Paraná. MSc. Thesis, Federal University of Paraná, Curitiba, Brazil. 129 pp.

Gomes, M. P.; Novelli, R.; Faria, V. V. and Zalmon, I. Z. (2001), Potencial Atrator de Peixes Ósseos em Recife Artificial no Litoral Norte do Estado do Rio de Janeiro, Brasil. Rev. Bras. Zool., 18 : (3), 779-792.

Harvey, C. J. (1998), Use of Sandy Beach Habitat by Fundulus majalis, a surf-zone fish. Mar. Ecol. Prog. Ser., 164, 307-310.

Hoefel, F. G. (1998), Morfodinâmica de Praias Arenosas Oceânicas: Uma Revisão Bibligráfica. Ed. UNIVALI - Itajaí, Brasil. 92 pp.

IBAMA (1986), Problemáticas Referentes à Pesca no Norte Fluminense. Serv. Pub. Fed. 7 pp.

IBAMA (1991), Pequena Análise do Levantamento Sócio-Cultural e Econômico das Comunidades pesqueiras de Atafona, Ilha da Convivência, Gargaú e S. João da Barra (sede), RJ. Serv. Pub. Fed. 17 pp.

Lasiak, T. A. (1984), Structural Aspects of the Surf Zone Assemblage at King's Beach, Algoa Bay, South Africa: Long-term Fluctuations. Est. Coast. Shelf Sci., 18 : (4), 459-483.

Lenanton, R. C. J.; Robertson, A. I. and Hansen, J. A. (1982), Nearshore Accumulations of Detached Macrophytes as Nursery Areas for Fish. Mar. Ecol. Prog. Ser., 9, 51-57.

Maes, J.; Taillieu, A.; Van Damme, P. A.; Cottenie, K. and Ollevier, F. (1998), Seasonal Patterns in the Fish and Crustacean Community of a Turbid Temperate Estuary (Zeeschelde Estuary, Belgium). Est. Coast. Shelf Sci., 47, 143-151.

Menezes, N. A. and Figueiredo, J. L. (1980), Manual de Peixes Marinhos do Sudeste do Brasil. IV Teleostei (3). Museu de Zoologia da Universidade de São Paulo, São Paulo. 96 pp.

Menezes, N. A. and Figueiredo, J. L. (1985), Manual de Peixes Marinhos do Sudeste do Brasil. V Teleostei (4). Museu de Zoologia da Universidade de São Paulo, São Paulo. 105 pp.

Modde, T. and Ross, S. T. (1981), Seasonality of Fishes Occupying a Surf Zone Habitat in the Northern Gulf of Mexico. Fish. Bull., 78 : (4), 911-923.
Modde, T. and Ross. S. T. (1983), Trophic Relationships of Fishes Occurring Within a Surf Zone Habitat in the Northern Gulf of Mexico. North.Gulf Sci., 6 : (2), 109-120.

Monteiro-Neto, C.; Blacher, C.; Laurent, A. A. S.; Snizek, F. N.; Canozzi, M. B. and Tabajara, L. C. C. de A. (1990), Estrutura da Comunidade de Peixes em Águas Rasas na Região de Laguna, Santa Catarina, Brasil. Atlântica, 12 : (3), 53-69.

Nash, R. D. M. and Santos, R. S. (1998), Seasonality in Diel Catch Rate of Small Fishes in a Shallow-Water Fish Assemblage at Porto Bim Bay, Fayal, Azores. Est. Coast. Shelf Sci., 47, 319-328.

Naughton, S. P. and Saloman, C. H. (1978), Fishes of the Nearshore Zone of St. Andrew Bay, Florida, and adjacent coast. Nor. Gulf. Sci., 2 : (1) 43-55.

Pessanha, A. L. M.; Araújo, F. G.; Azevedo, M. C. C. and Gomes, I. D. (2000), Variações Temporais e Espaciais na Composição e Estrutura da Comunidade de Peixes Jovens da Baía de Sepetiba, Rio de Janeiro. Rev. Bras. Zool., 17 : (1), 251-261.

Robertson, A. I. and Lenanton, G. J. (1984), Fish Community Structure and Food Chain Dynamics in the Surf-Zone of Sandy Beaches: The Role of Detached Macrophyte Detritus. Exp. Mar. Biol. Ecol., 84, 265-283.

Ross, S. T.; McMichael Jr.; R. H. and Ruple, D. I. (1987), Seasonal and Diel Variation in the Standing Crop of Fishes and Macroinvertebrates from a Gulf of Mexico Surf Zone. Est. Coast. Shelf Sci., 25, 391-412.

Sokal, R. R.; and Rohlf, F. J. (1981), Biometry: the Principles and the Practice of Statistic in Biological Research. $2^{\text {nd }}$ ed. W. H. Freeman and Co., San Francisco, USA. 859 pp.

Valentin, J. L. and Monteiro-Ribas, W. M. (1993), Zooplancton Community Structure on the EastSoutheast Brazilian Continental Shelf $(18-230 S$ latitude). Est. Cont. Shelf Sci., 13 : (4), 407-424.

Yañez-Arancibia, A. (1978), Taxonomía, Ecología y Estructura de las Comunidades de Peces en Lagunas Costeras con Bocas Efímeras del Pacifico del México. Centro Cienc. del Mar y Limnol., Univ. Nal. Auton. México, Publ. esp. 2. pp. 1-306.

Zar, J. H. (1999), Biostatistical Analysis. $3^{\text {rd }}$ ed. Prentice-Hall, New Jersey, EUA. 718 pp.

Received: September 28, 2001; Revised: June 13, 2002; Accepted: December 02, 2002. 